\title{
Involvement of a Novel TetR-Like Regulator (BdtR) of Bradyrhizobium diazoefficiens in the Efflux of Isoflavonoid Genistein
}

\author{
Fang Han, ${ }^{1}$ Xueqian He, ${ }^{1}$ Wenwen Chen, ${ }^{1}$ Haoyu Gai, ${ }^{1}$ Xuemei Bai, ${ }^{1}$ Yongxing He, ${ }^{1}$ Keisuke Takeshima, ${ }^{2}$ \\ Takuji Ohwada, ${ }^{2,+}$ Min Wei, ${ }^{1,+}$ and Fang $\mathrm{Xie}^{3}$ \\ ${ }^{1}$ State Key Laboratory of Grassland Agro-Ecosystems, Institute of Arid Agroecology, School of Life Sciences, Lanzhou \\ University, No. 222, South Tianshui Road, Lanzhou 730000, China \\ ${ }^{2}$ Department of Food Science, Obihiro University of Agriculture and Veterinary Medicine, Obihiro, Hokkaido 080-8555, Japan \\ ${ }^{3}$ National Key Laboratory of Plant Molecular Genetics, CAS Center for Excellence in Molecular Plant Sciences, Shanghai \\ Institute of Plant Physiology and Ecology, Chinese Academy of Sciences, Shanghai 200032, China
}

Accepted 10 September 2020.

\begin{abstract}
A wide variety of leguminous plant-released (iso)flavonoids, such as genistein, are potential inducers of the nodulation (nod) genes of endosymbiotic rhizobia for the production of Nod factors, which are vital signaling molecules for triggering the symbiotic process. However, these (iso)flavonoids are generally thought to be toxic to the bacterial partner to varying degrees. Here, a novel TetR-like regulator gene of the soybean symbiont Bradyrhizobium diazoefficiens USDA110, bdtR (systematic designation blr7023), was characterized. It was found to be rapidly and preferentially induced by genistein, and its mutation resulted in significantly increased expression of the neighboring bll7019-bll7021 genes, encoding a multidrug resistance efflux pump system, in the absence of this isoflavonoid. Then, the transcriptional start site of BdtR was determined, and it was revealed that BdtR acted as a transcriptional repressor of the above efflux system through the binding of an AT-rich operator, which could be completely prevented by genistein. In addition, the $\Delta$ bdtR deletion mutant strain showed higher accumulation of extracellular genistein and became less susceptible to the isoflavonoid. In contrast, the inactivation of BdtR led to the significantly decreased induction of a nodulation gene (nodY) independent of the expression of nodD1 and nodW and to much weaker nodulation competitiveness. Taken together, the results show that BdtR plays an early sensing role in maintaining the intracellular homeostasis of genistein, helping to alleviate its toxic effect on this bacterium by negatively regulating neighboring genes
\end{abstract}

${ }^{\dagger}$ Corresponding authors: M. Wei; weim@1zu.edu.cn and T. Ohwada; taku@obihiro.ac.jp

Funding: This work was supported by grants from the National Natural Science Foundation of China (31670504), National Key Laboratory of Plant Molecular Genetics and Japan Society for the Promotion of Science (24580099, 15K07346).

*The $\boldsymbol{e}$-Xtra logo stands for "electronic extra" and indicates there is a supplementary figure, a supplementary table, and supplementary text published online.

The author(s) declare no conflict of interest.

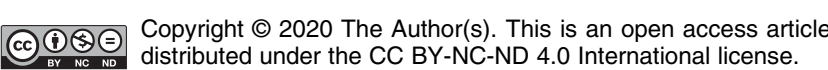

encoding an efflux pump system while being essentially required for nodule occupancy competitiveness.

Keywords: Bradyrhizobium diazoefficiens, isoflavonoid, genistein, efflux pump, nodulation competitiveness

Multidrug resistance (MDR) efflux pumps are a large transporter family consisting of cytoplasmic, periplasmic, and outer-membrane proteins, including the ATP-binding cassette (ABC) superfamily, multidrug and toxic compound extrusion family, small MDR family, resistance-nodulation-division (RND) superfamily, and major facilitator superfamily (Saier and Paulsen 2001). They have been reported to mediate the efflux of a variety of environmental toxic compounds (e.g., toxic metals, antibiotics, and solvents), which would consequently confer resistance on bacteria. Therefore, they are considered to play important ecological roles in the soil biosphere as well as plant-microorganism interactions (Martinez et al. 2009; Neyfakh 1997; Nikaido 1996). For example, some efflux pumps of the RND family have been shown to play a role in the colonization and persistence of bacteria in the host (Palumbo et al. 1998; Piddock 2006).

Rhizobia are soil bacteria that fix nitrogen (diazotrophs) after becoming established inside the root nodules of legumes. In Sinorhizobium meliloti, the deletion of the efflux system encoded by the $s m e A B$ gene results in increased sensitivity to a range of antibiotics, dyes, detergents, and plant-derived compounds as well as weaker nodulation competitiveness (Eda et al. 2011). In the same strain, Rossbach and associates (2014) described the structure and regulation of a TetR-like regulator encoded by the emrR gene, located adjacent to another efflux pump system gene, emrAB. Bradyrhizobium diazoefficiens, a symbiont of soybean (Glycine max L.), possesses more than 20 members of the RND family. The physiological function and genetic regulation of some efflux systems have been addressed, but their requirements for symbiotic interactions vary depending on the host plant (Krummenacher and Narberhaus 2000; Lindemann et al. 2010). An isoflavonoid (genistein and daidzein)-responsive putative TetR-like regulator, FrrA, has been characterized in $B$. diazoefficiens, but its deletion does not seem to affect the symbiotic performance of the bacterium (Wenzel et al. 2012). Genistein and daidzein are two main soybean-released isoflavonoids with similar structures and are 
known to serve as inducers of many clusters of symbiosis-related genes, such as nodulation (nod) genes, in B. diazoefficiens (Kosslak et al. 1987). These nod genes are responsible for the production of Nod factors, which are indispensable for triggering nodule formation on the roots of most host legume plants. On the other hand, many plant-derived (iso)flavonoids are thought to be involved in innate host defense and to exert toxic effects on associated bacteria. Parniske et al. (1991) reported that genistein could induce the resistance of $B$. diazoefficiens to soybean phytoalexins such as glyceollin, and such resistance was not altered in a strain with mutations in common nod $A B C$ genes. Most studies on the efflux systems of rhizobia have focused on changes in the sensitivity of bacteria to various toxic compounds (González-Pasayo and Martínez-Romero 2000). However, little is known about whether and how these efflux systems respond to legume plant-released (iso)flavonoids and their links with nodulation genes.

In a previous study, we identified a large genomic locus, BjG30 (at coordinates 7732147-7748456), of B. diazoefficiens USDA 110 because of its distinctive expression profile, by using a macroarray platform. BjG30 contains nine overlapping DNA probe fragments covering the bll7017 to bll7033 genes. All these DNA fragments were strongly and rapidly induced by genistein at $0.5 \mathrm{~h}$ postinduction (hpi) (Takeshima et al. 2013). In the same study, a strain lacking multiple genes from bll7019 to bll7021 (the $\triangle$ RND mutant strain used in this study) that putatively encode the components of an efflux pump system, showed defective nodulation and nitrogen-fixing abilities but exhibited increased susceptibility to genistein. In the present study, a putative TetR-like regulator gene, blr7023, was further characterized. The results show that blr7023 (designated $b d t R$ here, Bradyrhizobium diazoefficiens TetR-like regulator) is a transcriptional repressor of genes encoding the efflux system mentioned above and that the repression caused by the binding of BdtR to an operator can be completely relieved by the addition of genistein. The inactivation of BdtR results in greater accumulation of extracellular genistein, accompanied by the decreased susceptibility of cells to this flavonoid. In addition, the induction of nod genes in the B. diazoefficiens $\Delta \mathrm{bdtR}$ mutant strain is dramatically reduced compared with that in the wild-type (WT) strain B. diazoefficiens 110 . The present study provides novel insight into how $B$. diazoefficiens responds to the "double-edged sword" of the impacts of soybean-released (iso)flavonoids that are symbiotically beneficial (e.g., inducing nod genes) and simultaneously somewhat toxic to bacterial partners.

\section{RESULTS}

$b d t R$ was rapidly and preferentially induced by genistein.

Although previous experiments showed that BjG30 is a genistein-inducible region at $0.5 \mathrm{hpi}$, it is not known whether or how it responds to other soybean isoflavonoids, such as daidzein or soybean seed extract (SSE). Thus, we determined the expression profiles of $\mathrm{BjG} 30$ in the presence of these compounds, using the macroarray platform mentioned above and compared them with those of genistein. As shown in Supplementary Table S1, all nine DNA probe fragments were significantly induced 3.8 - to 57.3 -fold by genistein but not by daidzein (0.9- to 2.3-fold) at $0.5 \mathrm{hpi}$. Interestingly, the addition of daidzein greatly decreased their induction by genistein. Upon SSE treatment, four of the fragments showed induction of greater than 2.2-fold, a cut-off for significantly upregulated DNA fragments, but the levels were much lower than those induced by genistein. Furthermore, induction by genistein rapidly decreased to a very low level at $12 \mathrm{hpi}$ and even at $6 \mathrm{hpi}$ (data not shown). These results suggest that BjG30 is preferentially and rapidly induced by genistein at an early stage of the soybean- $B$. diazoefficiens 110 interaction. Even though the macroarray technique is not the most recent technology, the expression profile of $\mathrm{BjG} 30$ obtained here implies that the early molecular dialog between $B$. diazoefficiens 110 and soybean is rather complicated, because it is a large chromosomal region (e.g., with coverage of the genes from bll7019 to bll7033) and shows entirely different responses to these two structurally similar isoflavonoids.

Among the genes included within BjG30, the genes from bll7019 to blr7023 are organized as a cluster and are wellconserved in many B. diazoefficiens strains (Supplementary Table S1). According to the annotations compiled in the $B$. diazoefficiens USDA110 genome sequence database, the products of bll7019, bll7020, and bll7021 are predicted to be members of the AcrB/AcrD/AcrF family transporter, the efflux protein, and the HlyD family secretion protein, respectively. All these membrane proteins are highly homologous to the structural components of the bacterial MDR efflux pump system. Upstream of these three genes and in the opposite orientation, gene blr7023 encodes a putative TetR-type regulator. Interestingly, a putative bll7022 gene exists in the same orientation as the bll7019-bll7021 gene cluster and its homology with orthologs in other bacterial species shows that it probably encodes a sensor histidine kinase. This genetic organization suggests that the genes from bll7019-bll7022 are likely cotranscribed under the control of the BdtR protein (blr7023) and function as an operon. Considering that $\mathrm{BjG} 30$ is strongly induced by genistein, which has been assumed to be somewhat toxic to B. diazoefficiens 110 , we focused on the regulatory role of BdtR in the transcription of the nearby efflux system in response to soybean isoflavonoids in the present study.

Since the DNA probe fragments spotted on the membrane sheet of the macroarray system overlap and each contains more than one gene, the expression level of each gene could not be precisely deduced from the hybridization intensity of the corresponding DNA probe. Therefore, bdtR- and bl7022-lacZ transcriptional fusions were constructed to determine how the promoters of these genes respond to isoflavonoids. As shown in Figure 1, the activities of $b d t R$-lac $Z$ were significantly higher than the uninduced controls and rapidly decreased with time. At $2 \mathrm{hpi}$, their activity was approximately half that at $0.5 \mathrm{hpi}$. The activities of bll7022-lacZ were also mainly induced by genistein in a similar pattern; however, the levels were much lower overall than those of $b d t R$-lac $Z$. On the other hand, the activities of both $b d t R$ - and bll7022-lacZ were very low under daidzein treatment and were nearly equivalent to those of the blank control, which was only the isoflavonoid solvent dimethyl sulfoxide (DMSO). Since DMSO itself is a toxic compound, it seems that the activities of both $l a c Z$ were also stimulated to some degree. Furthermore, the addition of daidzein greatly inhibited the induction of the activities of both $b d t R$ - and bll7022-lacZ by genistein, suggesting that daidzein functioned antagonistically to genistein. The induction patterns of $l a c Z$ activities reported here were in agreement with the data from the macroarray analysis and confirmed that $b d t R$ is preferentially and rapidly induced by genistein at the early stage of soybean-B. diazoefficiens symbiosis.

\section{BdtR is a transcriptional repressor of adjacent genes encoding a MDR efflux system.}

To determine whether BdtR controls the transcription of its neighboring efflux pump system, $b d t R$ was first inactivated through the insertion of an $\Omega$ cassette ( $b d t R \Omega:: S m / S p c$ ) (Supplementary Text S1). Subsequently, the expression profile of BjG30 was captured in the presence of genistein at $0.5 \mathrm{hpi}$, using the same macroarray platform. As shown in Figure 2A, 
all three DNA probe fragments covering the genes from bll7019 to blr7023 were significantly induced by genistein at levels ranging from 15.7- to 22.1-fold in WT B. diazoefficiens 110 . In the $B$. diazoefficiens $\triangle \mathrm{RND}$ strain, in which most of the bll7019-blr7021 sequence was deleted, with the exception of brb21559 covering the single gene bll7019, two remaining DNA fragments were still induced 3.0- to 4.5-fold, which was still statistically significant. This is reasonable because each of these DNA fragments contains more than one gene that extends beyond the $5^{\prime}$ end of bll7021. Their expression was likely due to partial hybridization with ${ }^{33} \mathrm{P}$-cDNA fragments of genes other than bll7021. However, the hybridization intensity of these three DNA fragments was significantly higher in the $B$. diazoefficiens bdtR mutant strain than in the WT B. diazoefficiens 110 and $B$. diazoefficiens $\triangle \mathrm{RND}$ strains, regardless of whether genistein was added or not. Since B. diazoefficiens bdtR showed a severe growth defect, another in-frame deletion mutant, the $B$. diazoefficiens $\Delta \mathrm{bdtR}$ strain, was generated, and the relative expression levels of these four genes (bll7019 through bll7022) in response to genistein were quantified by real-time reverse transcription PCR (RT-PCR) (Fig. 2B). As expected, they were greatly and uniformly decreased in the $\Delta$ bdtR strain, consistent with the expression changes indicated by the macroarray analysis. The levels of these genes were partially restored in a genetic complementation strain (B. diazoefficiens HF18) of the B. diazoefficiens $\Delta \mathrm{bdtR}$ mutant, strongly suggesting that BdtR acts as a repressor to regulate the expression of efflux pump system operon genes bll7019 to bll7021.

The orientation of gene bll7022 is the same as that of bll7019-bll7021, and they are simultaneously induced by genistein as described above, suggesting that they are cotranscribed under the control of common operators, which are presumably located within the intergenic region between bll7022 and blr7023. Therefore, the existence of BdtR-DNA binding sites (or operators) within this region and whether binding at the sites can be affected by the addition of isoflavonoids were first determined in an electrophoretic mobility shift assay (EMSA). As shown in Figure 3A, as the concentration of the BdtR protein increased, two differently migrating bands were detected, suggesting that there are two operators with different binding strengths for the BdtR protein, which probably functions as a dimer. When genistein was added at concentrations from $250 \mu \mathrm{M}$ to $2 \mathrm{mM}$, these two shifted-DNA bands completely vanished, indicating that genistein strongly interferes with BdtR-DNA complex formation. However, unlike genistein, which produced clear-cut results, the addition of daidzein did not substantially affect the formation of the BdtR-DNA complex. The shifted-DNA band seemed to show an indistinct decrease in intensity at higher concentrations (e.g., 1 to $2 \mathrm{mM}$ ) (Fig. 3B). Furthermore, the prevention of BdtR-DNA binding by genistein was affected very little by the addition of daidzein (Fig. 3C). The results reported here suggest that genistein is the main compound that preferentially affects the binding of BdtR to two operators, while daidzein may block their binding very weakly. These EMSA data provide a clear and more detailed molecular mechanism to explain the results of the promoter activity assay and macroarray analysis.

A putative 27-bp-long palindromic sequence (PS) potentially serving as an operator was found to be located $73 \mathrm{nt}$ upstream of the putative start codon of BdtR (Fig. 4). To evaluate its conservation, the alignment of the nucleotide sequences around this PS with those of neighboring genes in other genomes showing the same top COG (clusters of orthologous groups of proteins) hit in other strains of B. diazoefficiens was conducted. As shown in Figure 5A, this PS element is highly conserved among the $B$. diazoefficiens strains, indicating that it is not dispensable for physiological function and ecological behavior. Subsequently, a DNase I footprinting assay was performed to confirm the presumed operator (Fig. 5B). The result revealed that there is only one BdtR-binding site in the bll7022-bdtR intergenic region, and its nucleotide sequence fully matched the predicted operator sequence in Figure 5A, except for five nucleotide acids (a $\mathrm{T}$ before at the $5^{\prime}$ end and TTGT before the $3^{\prime}$ end). Since it is symmetrical, the two shifted bands in Figure 3 are likely due to the differences in the affinity of BdtR, which probably acts as a dimer, for each half of this operator. Whether this palindromic region acts as a BdtR-binding site was further determined by EMSA. Figure 5C shows that, as the amount of the BdtR protein increased, the movements of artificially synthetized PSs were gradually retarded due to increased binding. This was further proven, using a DNA fragment containing the $b d t R$-bll7022 intergenic region but lacking the operator sequence. As shown in Figure 5D, the deletion of the operator resulted in the complete disappearance of the two BdtR-DNA complexes. The BdtR protein clearly functions as a repressor binding to this operator, sterically interfering with the binding of RNA polymerase to the promoters of the $b d t R$ and $b l l 7022$ genes and consequently regulating their transcription.

Interestingly, this region is AT rich, which may facilitate the initial separation of the DNA strands for RNA synthesis. Bioinformatics analysis demonstrated that it overlapped with the
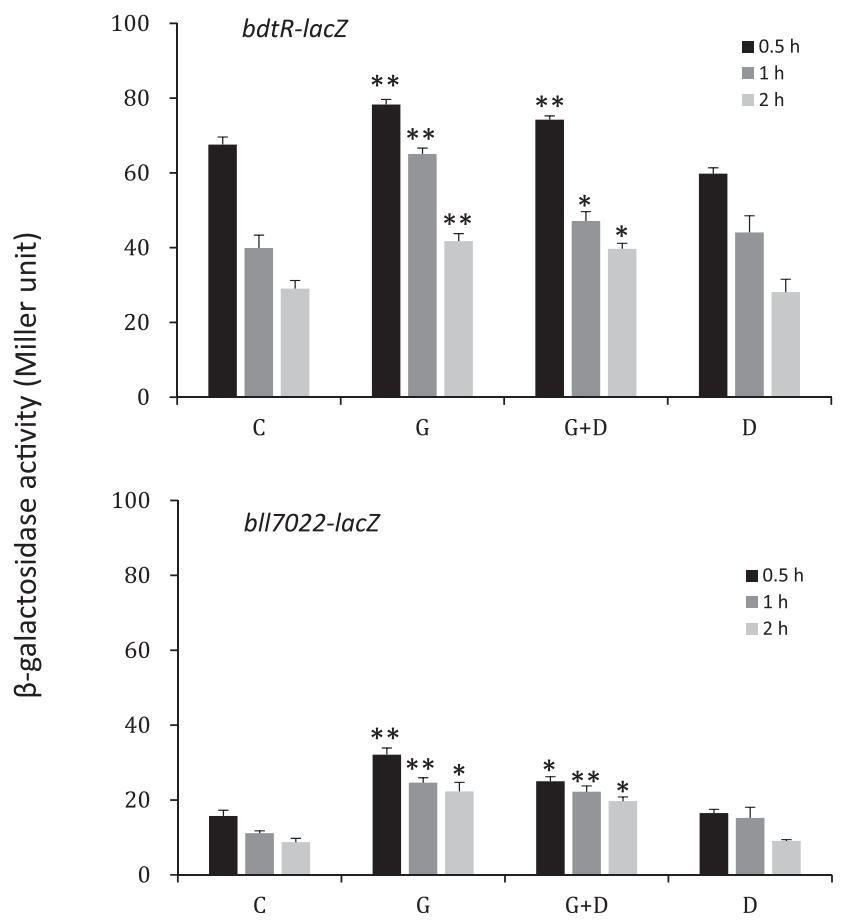

Isoflavonoid

Fig. 1. Promoter activities of the $b d t R$ and $b l l 7022$ genes in response to $5 \mu \mathrm{M}$ soybean isoflavonoids. $\mathrm{C}=$ control $=$ only the isoflavonoid solvent dimethyl sulfoxide (DMSO), $\mathrm{G}=$ genistein, $\mathrm{D}=$ daidzein, $\mathrm{G}+\mathrm{D}=$ mixture of $\mathrm{G}$ and D. Bradyrhizobium diazoefficiens strains HQ30 and HQ31 carrying pKS818::bll7022-lacZ and pKS819::bdtR-lacZ were grown in yeast extract-mannitol (YEM) medium to the logarithmic phase and were diluted with fresh YEM to an optical density at $600 \mathrm{~nm}=0.1$. After the addition of the corresponding isoflavonoids, $\beta$-galactosidase activities were assayed at different timepoints from at least three independent cultures. Error bars represent the standard error of the mean. Columns labeled with asterisks represent values that are significantly higher than the values obtained in the control (uninduced strains). Two asterisks (**) indicate $P<0.01$, one (*) indicates $P<0.05$. 
-10 element of the $b d t R$ promoter. Moreover, the transcriptional start site of bll7022, which was determined by rapid amplification of cDNA ends (5'-RACE) in this study (Supplementary Text S1), is located within this PS (Fig. 4). Such overlapping features of regulatory elements with operators suggest a significant contribution of this operator to the

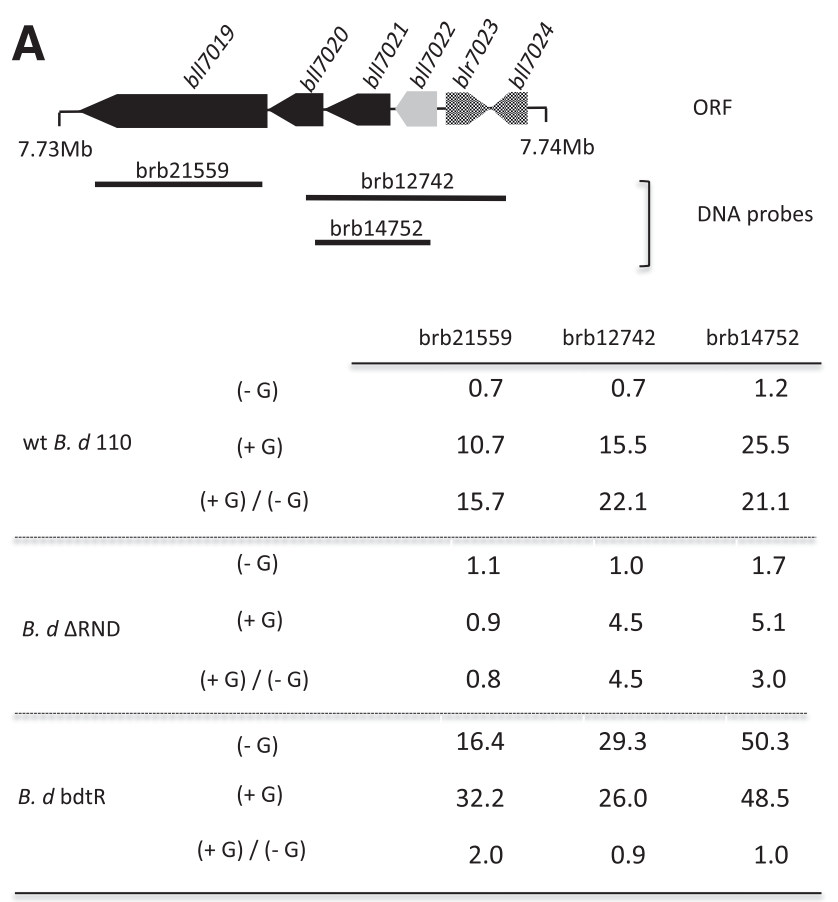

G: genistein

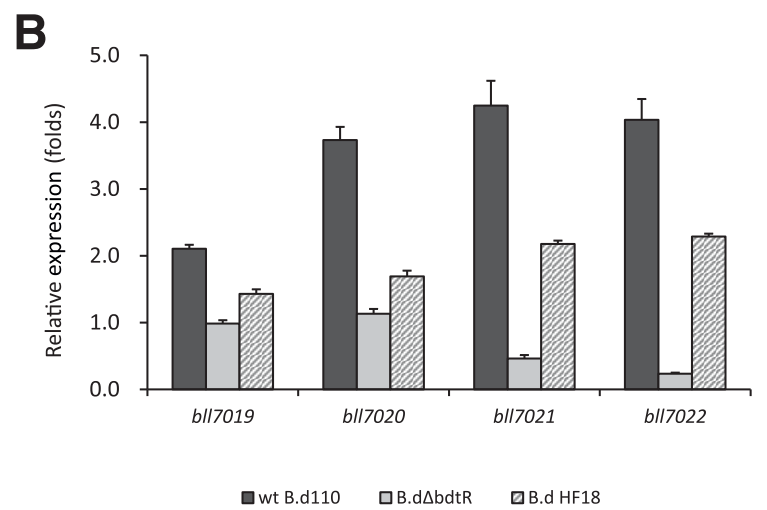

Fig. 2. A, The comparative expression of three DNA probe fragments around bll7019-blr7023 between Bradyrhizobium diazoefficiens wild-type (WT) strain 110, B. diazoefficiens $\triangle \mathrm{RND}$, and B. diazoefficiens bdtR in response to genistein at $0.5 \mathrm{~h}$ postinduction (hpi). The expression of the start and end positions of the DNA probe fragments on the chromosome was assessed, using a macroarray platform as previously described (Wei et al. 2008). Briefly, a total of 3,962 overlapping DNA probe fragments that were individually amplified by PCR from an M13 library with coverage of approximately $98.7 \%$ of the whole genome of WT $B$. diazoefficiens 110 were spotted onto a sheet of membrane for hybridization with ${ }^{33} \mathrm{P}$-labeled cDNA, which was synthesized by reverse transcription from messenger RNA isolated from cells in the presence $(+G)$ or absence $(-G)$ of inducer genistein at 0.5 hpi. (+G/-G) indicates the expression change (fold) in response to genistein. Values in the table indicate the normalized digital hybridization intensity of the DNA probes against the total for all DNA fragments. B, Relative expression of genes from bll7019 to bll7022 in WT B. diazo-

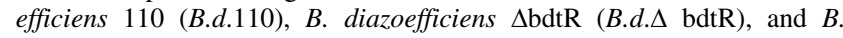
diazoefficiens HF18 (B.d.HF18) in the presence of $5.0 \mu \mathrm{M}$ genistein at 0.5 hpi. Values represent the mean of two independent experiments with the standard error. regulation of bll7022 as well as $b d t R$ itself and can possibly explain the weaker activity of bll7022-lacZ compared with that of bdtR-lacZ (Fig. 1).

\section{Inactivation of BdtR resulted in higher accumulation of extracellular genistein and the low susceptibility of cells to genistein.}

It has been recognized that the multidrug efflux pumps of many bacteria are involved in resistance to various antibiotics and toxic compounds. If it is true that $\mathrm{BdtR}$ is a negative transcriptional regulator of bll7019-bll7021, as mentioned above, inactivation would alter the normal efflux process in the bacterium, resulting in altered cellular susceptibility toward genistein, which is itself toxic to B. diazoefficiens 110 . Therefore, the extracellular genistein concentration of the B. diazoefficiens $\Delta$ bdtR mutant was monitored over time (Fig. 6A). It gradually decreased in all three strains, indicating that the intake process was unlikely to be damaged. However, the genistein content outside the $\Delta \mathrm{bdtR}$ mutant decreased more slowly than that in WT B. diazoefficiens 110 and the genetic complementary strain $B$. diazoefficiens HF18. This can be explained by the fact that the inactivation of BdtR relieved its repression of the transcription of bll7019-bll7021 and, consequently, the efflux of genistein outside of the cell might have been promoted. This was supported by the evidence demonstrating that the $B$. diazoefficiens bdtR strain showed higher expression of the DNA fragments containing the bll7019-bll7021 genes than WT B. diazoefficiens 110 (Fig. $2 \mathrm{~A})$. If the efflux of genistein in the $\Delta \mathrm{bdtR}$ mutant were enhanced, its toxic effect on the cells would be reduced, resulting in lower susceptibility of cell growth to the effect. Thus, the growth response of the $\Delta \mathrm{bdtR}$ mutant to genistein and daidzein was investigated. As shown in Figure 6B, the growth of WT B. diazoefficiens 110 was greatly inhibited by $100 \mu \mathrm{M}$ genistein, maintaining the optical density at $600 \mathrm{~nm}$ $\left(\mathrm{OD}_{600}\right)$ below 0.4 , while the $\Delta$ bdtR mutant strain continued to grow, reaching an $\mathrm{OD}_{600}$ of approximately 0.6 by the end of the experimental period. Interestingly, daidzein at $100 \mu \mathrm{M}$ inhibited the growth of WT B. diazoefficiens 110 to the same degree as genistein only in the early growth phase (e.g., until day 7), indicating that daidzein is less toxic than genistein. However, such inhibition was completely relieved in the $\Delta$ bdtR mutant because it showed rapid growth from the beginning of the experiment. The findings that strain $B$. diazoefficiens HF18 restored the physiological behavior of the $\Delta$ bdtR mutant and that the $B$. diazoefficiens $\Delta \mathrm{RND}$ mutant strain was more susceptible to genistein than the $\Delta$ bdtR strain (Takeshima et al. 2013) suggest that the deletion of BdtR is responsible for the activation of its adjacent efflux system to pump isoflavonoids outside the cells.

\section{The inactivation of BdtR significantly reduced the induction of nodulation genes.}

Genistein and daidzein show the potential to induce the expression of nodulation genes in B. diazoefficiens 110 (Banfalvi et al. 1988). Therefore, the effect of BdtR on the induction of nodulation genes was determined by using nodY, an important structural nodulation gene. Compared with WT B. diazoefficiens 110 , the induction of $\operatorname{nod} Y$ was significantly decreased in the mutant $B$. diazoefficiens $\Delta \mathrm{bdtR}$ strain in the presence of varying concentrations of genistein (Fig. 7A). We noted that the $B$. diazoefficiens $\Delta$ bdtR strain was more resistant to the tetracycline (Tc) antibiotic than WT B. diazoefficiens 110 (data not shown), implying that BdtR is also involved in the negative regulation of Tc efflux. Since the nodY-lacZ fusion was generated in a broad-host-range plasmid with Tc resistance, it was inferred that $\operatorname{nod} Y$ induction levels were probably affected by the plasmid 
copy number induced by the selective pressure from Tc. To confirm this, the relative expression level of the nodY gene in strain $B$. diazoefficiens $\Delta$ bdtR was quantified using real-time RTPCR and the results showed that, similar to nodY-lacZ activity, its relative expression levels at 6 and 12 hpi were significantly decreased (Supplementary Fig. S1). Then, the induction of nodY by genistein, daidzein, or SSE was determined in both strains. Overall, the induction of nod $Y$ was decreased in the $\Delta$ bdtR mutant strain to a much greater degree in the presence of genistein (Fig. 7B). It has been solidly demonstrated that in B. diazoefficiens, NodD1, and NodW are essentially required for the full induction of the nod gene by soybean isoflavonoids (Göttfert et al. 1989; 1990; Loh et al. 1997). Therefore, whether the induction of nodY was affected by the defective expression of these two genes, which might result from the inactivation of BdtR, was investigated, using chromosome-integrated nodDl- and nodW-lacZ transcriptional fusions. As shown in Figure 7C, the inductions of both genes were not substantially altered except for that in nodD1 at $0.5 \mathrm{hpi}$, indicating that the dramatically decreased induction of nod $Y$ in the $\Delta$ bdtR mutant strain was due to the disturbance of intracellular genistein levels rather than the effects of the nodD1 and $\operatorname{nod} W$ genes. The results reported here suggest that BdtR plays an important regulatory role in maintaining intracellular genistein homeostasis, rather than the direct linkage of signal transduction with the regulation of nodulation genes.

\section{Deletion of $b d t R$ weakened the nodulation competitiveness of $B$. diazoefficiens.}

The efflux system plays an important role in the extrusion of toxic substances to the environment and plant-released flavonoids, which might be beneficial for bacteria to establish successful interactions with host plants. It is tempting to speculate that the deletion of $b d t R$ would affect the competitive nodulation ability of $B$. diazoefficiens. Therefore, the co-inoculation experiments in which GusA-marked WT B. diazoefficiens 110 (B. diazoefficiens A20) was mixed with each of the WT $B$. diazoefficiens $110, \Delta \mathrm{bdtR}$ mutant, and $B$. diazoefficiens HF18 strains at a ratio of $1: 1$, followed by inoculation onto Glycine $\max$ cv. Suinong 4. As shown in Figure 8, the proportion of nodule occupancy induced by the $\Delta$ bdtR mutant was significantly less than that induced by $B$. diazoefficiens A20. However, WT B. diazoefficiens 110 and B. diazoefficiens HF18 induced nodule occupancy to approximately the same extent as B. diazoefficiens A20. The results reported here suggest that the inactivation of BdtR greatly weakened the nodulation competitiveness of B. diazoefficiens 110 .

\section{DISCUSSION}

BjG30 is a large distinctive genistein-responsive expressed region identified on a macroarray platform at $0.5 \mathrm{hpi}$. Although
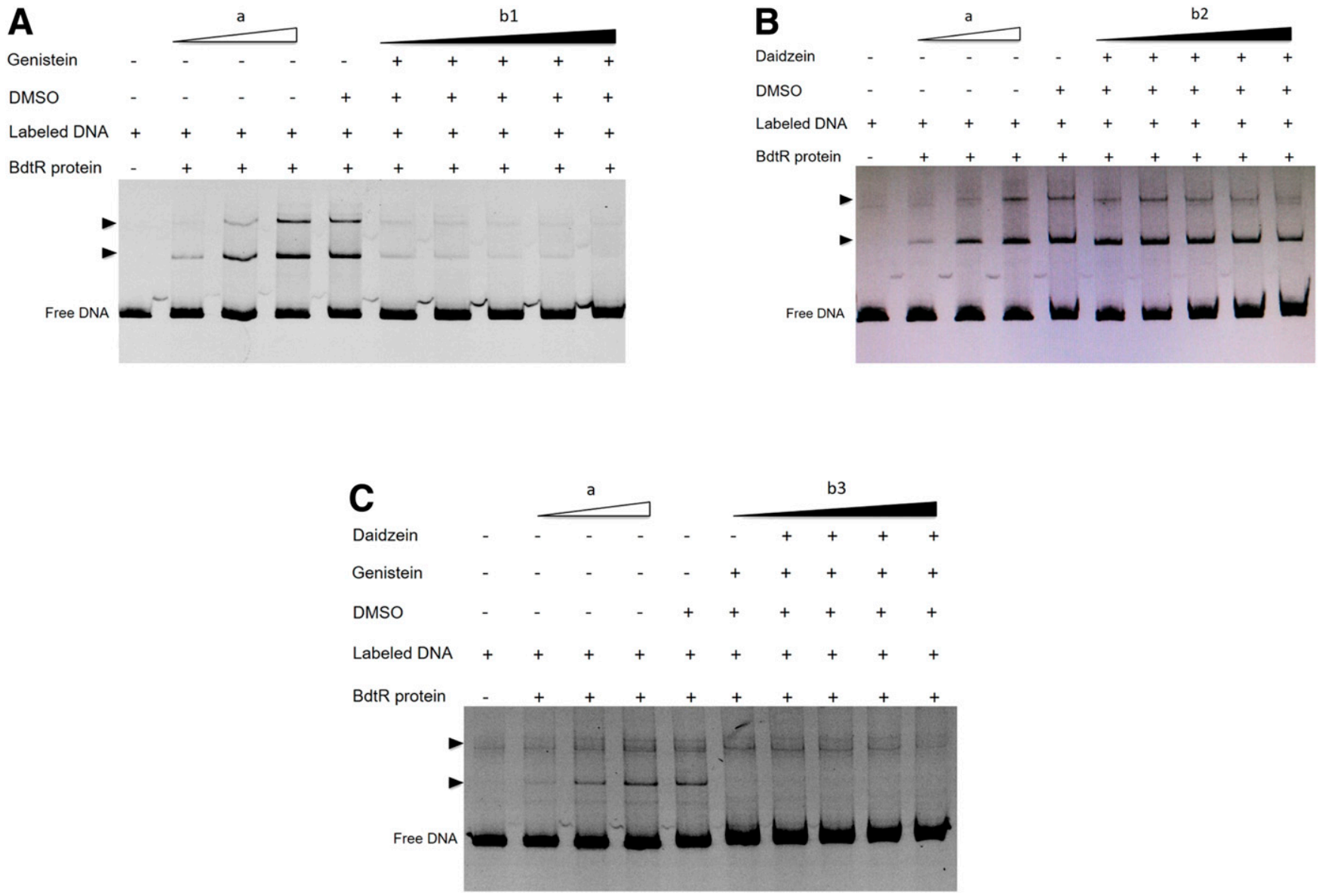

Fig. 3. Electrophoretic mobility shift assay with purified BdtR protein, a Cy5-labeled 234-bp-long PCR product encompassing the intervening region of bll7022-bdtR and the soybean isoflavonoids A, genistein, B, daidzein and $\mathbf{C}$, the mixture of both. The presence ( +$)$ or absence $(-)$ of isoflavonoids, the dimethyl sulfoxide (DMSO) solvent, and the BdtR protein is indicated above the gel image. In A and B, genistein and daidzein concentrations were, from left to right, as indicated by the wedge (b1 and b2), 250, 500, $750 \mu \mathrm{M}$ and 1 and $2 \mathrm{mM}$, respectively. In C, daidzein concentrations were, from left to right, as indicated by the wedge (b3), 0, 250, $500 \mu \mathrm{M}$ and 1 and $2 \mathrm{mM}$, respectively, while the genistein concentration was maintained at $2 \mathrm{mM}$. Throughout the experiment, $11 \mathrm{ng}$ of the Cy5-labeled PCR products and $848 \mathrm{ng}$ of the BdtR protein were used, except in three wells containing increasing amounts of 106,424 , and 848 ng, as indicated by wedge a. Arrowheads point to the DNA-protein complex. It is noteworthy that in B and C, the addition of daidzein, particularly at high concentrations, seems to cause an imperceptible decrease in the DNA-protein complex. 
this platform is not the most recent technology, the obtained expression profile is still useful for narrowing the functional genomic locus that responds to soybean-released isoflavonoids during symbiotic development. The distinctive expression profile of BjG30 suggests that the early molecular dialog between soybean and $B$. diazoefficiens is probably more complicated than the suggested isoflavonoid (soybean)-nod gene (bacteria) interaction. In the present study, we mainly analyzed the regulatory role of Blr7023 (BdtR) in the transcription of the adjacent efflux pump system encoded by the genes from bll7019 to bll7021 in response to soybean isoflavonoids. BdtR may be involved in the global transcription regulation of other genes within or outside of BjG30. For example, an oligonucleotide sequence (AAATAAACTACACCGTATATT) that is highly homologous to the operator examined in the present study was intriguingly found to be located within the intergenic regions of bll7025 and blr7026. However, both products are annotated as hypothetical proteins. An insertional mutant $b d t R$ strain was first generated for this study. Unfortunately, it showed severe growth defects, which was likely caused by polar effects. Additionally, the macroarray platform was useful for capturing the genome-wide expression portrait but could not provide any information on individual genes. Thus, except for the clone fragments located around $b d t R$ (Fig. 2A), no other data from the macroarray analysis were presented in this study. The global role of BdtR in the early events of symbiosis needs to be studied further.

To our knowledge, there have been no other reports about the regulatory determinants that respond to isoflavonoids so early in this bacterium. According to RhizoBase, B. diazoefficiens USDA 110 has 58 TetR-type regulators, including Blr4322 (FrrA), which was characterized by Wenzel et al. (2012). FrrA is also encoded by a gene located near genistein-inducible genes encoding an FreABC efflux system. FrrA and BdtR are similar in some aspects. They are both repressors of their neighboring efflux system, and this repression can be relieved by genistein. However, their expression patterns are different. According to our macroarray data, DNA fragments covering

\section{Bl17022 \\ GGGCACGACATATTCGCTCTCTCCTGAGGTCGACCGGAC}

GATCGCAAGGCCAATGCACACAGGTTTGCGGCCTCGAC

TGTCCGGGAGGTGCCGCCGCTGGTCCTGATGGACGTCG

10

CGGCGATTAAATAAACTATACCGTATATTTTCTTTTTGTTG
CCTTTTCCGGCCCGCCATGTCAAGTGCGATCTGCGCGCG
CACAGACTCGTGACTGGAGGATCGACGGATGACCAAG

\section{AA}

Fig. 4. Putative regulatory elements within the intergenic region between the $b l l 7022$ and $b l r 7023$ (bdtR) genes. All putative regulatory elements of genes bll7022 and blr7023 are indicated in blue and red, respectively. Arrows indicate the annotated translational start codons of bll7022 and blr7023, which are each preceded by an AG-rich Shine-Dalgarno (S-D)-like sequence. Putative 35/-10 promoter elements for gene blr 7023 , which were predicted by the BPROM program (Solovyev and Salamov 2011), are outlined with dotted lines. One palindromic sequence serving as an operator for BdtR is underlined in black. The transcriptional start sites of two genes that were identified in the present study are marked with asterisks. the $b d t R$ locus were preferentially and transiently induced by genistein to a very high level (15.7- to 57.3 -fold) at $0.5 \mathrm{hpi}$ (Supplementary Table S1), while those covering the frrA locus were significantly and constitutively induced by both genistein and daidzein within $12 \mathrm{hpi}$ by no more than 10 -fold. In addition, the inactivation of BdtR did not affect the induction of frrA by genistein (data not shown). This indicates that the responses of TetR-type regulators in B. diazoefficiens to soybean isoflavonoids are versatile and that there may exist no crosstalk between FrrA and BdtR. Thus, as far as the induction time and levels are concerned, BdtR is apparently a novel genisteinresponsive regulator of $B$. diazoefficiens at the early stage of symbiosis.

BdtR functioned as a repressor to regulate the expression of genes from bll7019 to bll7021 by binding to the intergenic region between bll7022 and $b d t R$, and this repression could be greatly relieved by the addition of genistein (Fig. 3A). However, we do not know whether the transcription of bll7019bll7021 is directly regulated by BdtR or is mediated by Bll7022. In addition, the induction of genes by genistein was greatly inhibited by daidzein, indicating that daidzein probably also interferes with the BdtR-DNA complex in a manner that is antagonistic to genistein. The EMSA results revealed that daidzein likely also impaired the BdtR-DNA complex at higher concentrations (Figs. 3B and 4C). However, the changes in the intensity of the shifted band were not clear-cut, and more sensitive methods are needed to further address this issue. Interestingly, daidzein is structurally similar to genistein, only lacking a hydroxyl group at the five-carbon position. A variety of other (iso)flavonoids have been used to investigate the promoter activities of genes by using bll7022- and bdtR-lacZ (data not shown), but the results have not indicated the structural characteristics of these compounds related to how they differentially interact with BdtR. Moreover, it should be noted that this efflux system operon is probably less active in the natural environment, since its expression levels were not substantially stimulated by SSE (Supplementary Table S1). At least daidzein, among the components of SSE, was shown to inhibit the induction of this operon. This may hint at biological significance, because some genetic elements of host-associated bacteria, such as quorum-sensing systems, have been reported to be strongly induced by the autoinducer signal but can also be interfered with by host plant-derived mimics (Gao et al. 2003; González and Keshavan 2006).

The deletion of $b d t R$ resulted in a significant reduction in $n o d Y$-lac $Z$ activity in response to varying concentrations of genistein (Fig. 7A). Subsequently, we revealed that the induction of nodDl and nodW was not affected by the inactivation of BdtR, suggesting that the decrease in the nod $Y$ gene was caused by a disorder of intercellular homeostasis of genistein. This conclusion is partially supported by evidence showing that a $b d t R$ mutation resulted in the abnormal accumulation of extracellular genistein (Fig. 6A). In addition, no nod box-like sequence was found in the intergenic region of this efflux system gene operon. The nod box is the binding site of NodD, a LysR-type regulator, which activates nodulation genes in the presence of flavonoids (Schlaman et al. 1998). However, it has been reported that the induction of nodY is cell population density-dependent and can be repressed at a high cell population density via NodD2 (Loh et al. 2002). The possibility that BdtR is also involved in the efflux of quorumsensing autoinducers cannot be ruled out. Increasing evidence suggests that some efflux pumps are involved in the elimination of endogenous toxic compounds generated by bacterial metabolism (Neyfakh 1997). For instance, in Pseudomonas aeruginosa, the MexGHI-OpmD MDR pump appears to be implicated in the extrusion of thranilate, a toxic precursor of 
A

USDA110

elkanii

huanghuaihaiense

CP.3

USDA122

WSM2254

USDA38

WSM471

USDA124

SEMIA5080

consensus sequence
Symmetrical operator halves

1

----CGCGGCGA-------TTAAATAAACTATACCGTATATTTTCTTT--TTGTTGCCT

GACGGGCAAAGAAAGGCAATAAAAAGAAAATATACGGTATAGTTTATTAAATCGTCAGCC

GGCTCGCGAAGAAAGGCAACAAAAAGAAAATATACGGTATAGTTTATTATTTCGTCAG--

GGCTCGCGAAGAAAGGCAACAAAAAGAAAATATACGGTATAGTTTATTATTTCGTCAG--

GGCGGGCCGGAAAAGGCAACAAAAAGAAAATATACGGTATAGTTTATTTAATCGCCGC--

GGCGGGCGGGAAAAGGCAACAAAAAGAAAATATACGGTATAGTTTATTTAATCGCCGC--

GCCCGGTGGGAAAAGGCAATAAAAAGAAAATATACGGTATAGTTTATTTAATCGTCGC--

GCTCCGCCGAAAAAGGCAACAAAAAGAAAATATACGGTTTAGTTTATTTAATCGTCGC--

GGGGCCTGACGA-------AATAATAAACTATACCGTATATTTTCTTT--TTGTTGCCT

----CGCGGCGA-------TTAAATAAACTATACCGTATATTTTCTTT--TTGTTGCCT
B

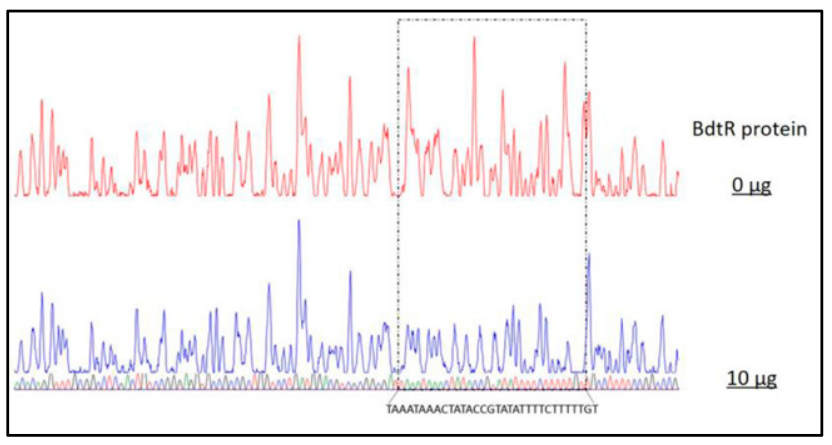

C PS

BdtR protein
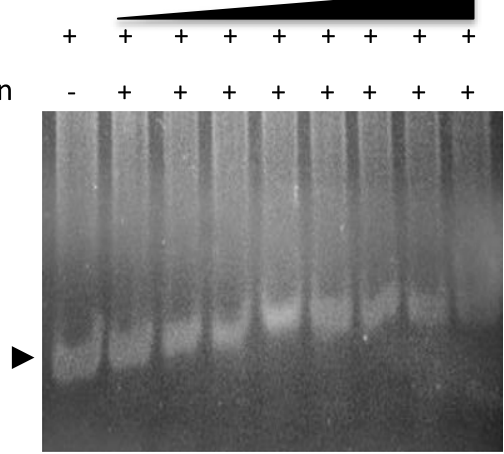

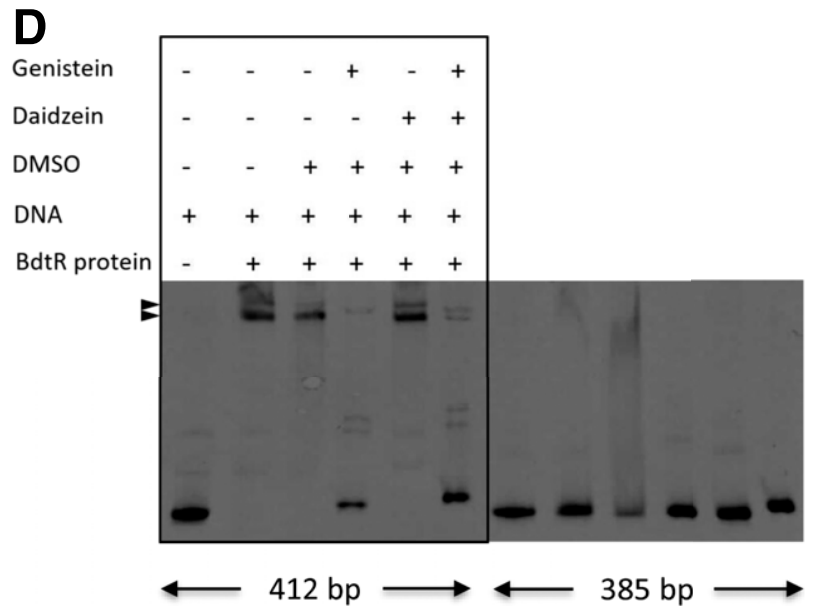

Fig. 5. A, Alignment of nucleotide sequences around the 27-bp-long palindromic sequence (PS) with those of neighboring genes in other genomes with the same top COG (clusters of orthologous groups of proteins) hit from some Bradyrhizobium diazoefficiens strains. The consensus sequence illustrates only the nucleotides with $100 \%$ identification. B, Identification of the BdtR-binding site in the bll7022-bdtR intergenic region by a DNase I footprinting assay. The probe used was labeled with FAM dye. The regions protected by BdtR (BdtR-binding site) are boxed with a dotted line. C, Electrophoretic mobility shift assay (EMSA) with purified BdtR and an artificially synthetized 27-bp-long PS. Different amounts of protein, as indicated by the wedge (3.3, 4.3, 5.4, 6.5, 7.6, 8.7, 9.8 , and $10.9 \mu \mathrm{g}$ ) were incubated with $150 \mu \mathrm{M}$ PS and were then loaded into a $12 \%$ polyacrylamide gel, subjected to electrophoresis, and stained with ethidium bromide. D, EMSA with purified BdtR and a 412-bp-long PCR fragment used for the construction of pKS818 (left box) and the same DNA fragment lacking PS (385 bp, right part). BdtR protein: $2.4 \mu \mathrm{g}$; DNA fragment: $300.0 \mathrm{ng}$; genistein: $1.0 \mathrm{mM}$; daidzein: $1.0 \mathrm{mM}$. The reaction solution was loaded into a $6 \%$ polyacrylamide gel for electrophoresis, which was stained with ethidium bromide. The two arrowheads point to the DNA-protein complexes. 
the Pseudomonas quinolone signal (Aendekerk et al. 2005; Sekiya et al. 2003).

The deletion of $b d t R$ significantly weakened the nodulation competitiveness of B. diazoefficiens (Fig. 8). This was unexpected because the $\Delta$ bdtR strain showed decreased sensitivity to the toxic isoflavonoids used in the study. Evidence showed that the (iso)flavonoid contents of the soybean plant vary in the different tissues and the developing stages and the varieties of soybean. The (iso)flavonoid profiles of soybean can also be affected by environmental factors such as the addition of nitrogen fertilizer and inoculation of rhizobia and microbial elicitors (pathogenic or beneficial bacteria). Graham (1991) detected about $400 \mathrm{nM}$ genistein in the seed exudates of soybean during an imbibition of $24 \mathrm{~h}$, but this concentration did not reach a saturation state. Indeed, this concentration is lower than we used in the study. Therefore, recently, we investigated the growth of WT $B$. diazoefficiens 110 strain in the presence of genistein of $0.5,1.0$, and $10 \mu \mathrm{M}$. Unexpectedly, the growth of bacteria was slightly stimulated rather than inhibited at the two former concentrations. A possible interpretation is that the concentration of $400 \mathrm{nM}$ just represents a mean value of genistein secreted from the soybean seed. The root macroenvironment that the B. diazoefficiens 110 inhabits may be in heterogenicity. For example, it is known that the root tip of a plant is a very active site to secrete many kinds of plant-derived metabolites. We do not know how much genistein is secreted from the soybean root hair, where $B$. diazoefficiens 110 initially interacts with the legume plant. However, the concentration of soybean-released genistein in some root macroenvironments is perhaps far

A
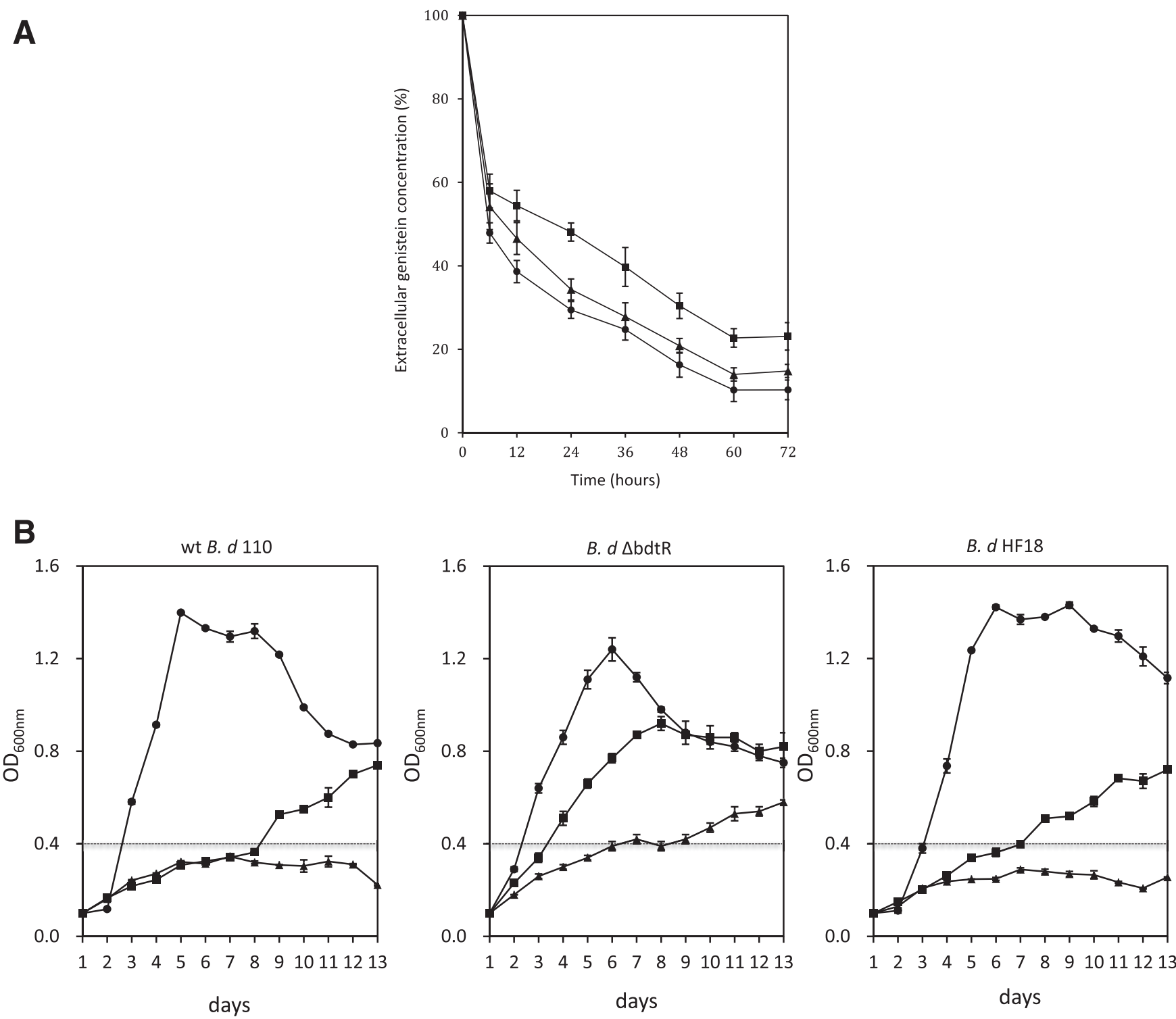

Fig. 6. A, Percentage of extracellular genistein concentration during the growth of wild-type (WT) Bradyrhizobium diazoefficiens 110 (triangles), B. diazoefficiens $\Delta \mathrm{bdtR}$ (squares), and B. diazoefficiens HF18 (dots) in yeast extract-mannitol (YEM) broth medium. Cells of the three strains were grown to logarithmic phase (optical density at $600 \mathrm{~nm}\left[\mathrm{OD}_{600}\right]=0.4$ to 0.6 ) and were diluted to $\mathrm{OD}_{600}=0.1$. Then, genistein was added to a final concentration of $10.0 \mu \mathrm{M}$. The genistein concentration in the culture supernatant was measured over time via high-pressure liquid chromatography. The genistein content was normalized to the total amount of cellular protein, and the percentage was calculated based on the initial concentration. B, The growth pattern of WT $B$. diazoefficiens 110 (B. $d$ 110), the mutant strain B. diazoefficiens $\Delta \mathrm{bdtR}(B . d \Delta \mathrm{bdtR})$, and the genetically complementary strain $B$. diazoefficiens $\mathrm{HF} 18$ (B. $d$ HF18) in YEM broth medium with a final concentration of $100 \mu \mathrm{M}$ isoflavonoids. Control (dots), daidzein (squares) and genistein (triangles). The horizontal dotted line indicates the $\mathrm{OD}_{600}$ value when the growth of WT B. diazoefficiens 110 was completely inhibited. The results represent the mean of data from two independent experiments, and the error bars indicate the standard error. 
higher than $400 \mathrm{nM}$ and may have a toxic effect on B. diazoefficiens 110 . Moreover, the $B$. diazoefficiens $\Delta$ bdtR strain may still be susceptible to other unknown phytoalexin compounds, such as glyceollin, that are released from soybean in the natural rhizosphere niche. Another possibility is that the weaker nodulation competitiveness of the $\Delta$ bdtR mutant strain was due to the decreased induction of nod genes. SSE is a mixture of ethyl alcohol-extracted compounds that is expected to be more similar to the naturally occurring spectrum of hostderived agents that $B$. diazoefficiens encounters at the early stage of symbiosis. As shown in Figure 7C, the induction of the $\operatorname{nod} Y$ gene by SSE in the $\Delta b d t R$ mutant strain was still decreased to some extent compared with that in the wild-type strain.
From an evolutionary perspective, symbiosis islands are large mobile genetic elements (genomic islands [GIs]) that are thought to be acquired during adaptive evolution through horizontal gene transfer (Dobrindt et al. 2004). In addition to symbiosis islands, 14 small GIs have been predicted on the chromosome of B. diazoefficiens 110 (Kaneko et al. 2002). However, the efflux system studied herein does not belong to any of these GIs, suggesting that it is likely an evolutionarily ancient element. The evidence showing that the induction of nodD1 and nodW was independent of BdtR also seems to support this deduction. The present study provides novel insight into the adaptation mechanism underlying the sensing and response of symbiotic bacteria to the "double-edged sword" (toxic effect vs. symbiotic signal) of the impacts of leguminous
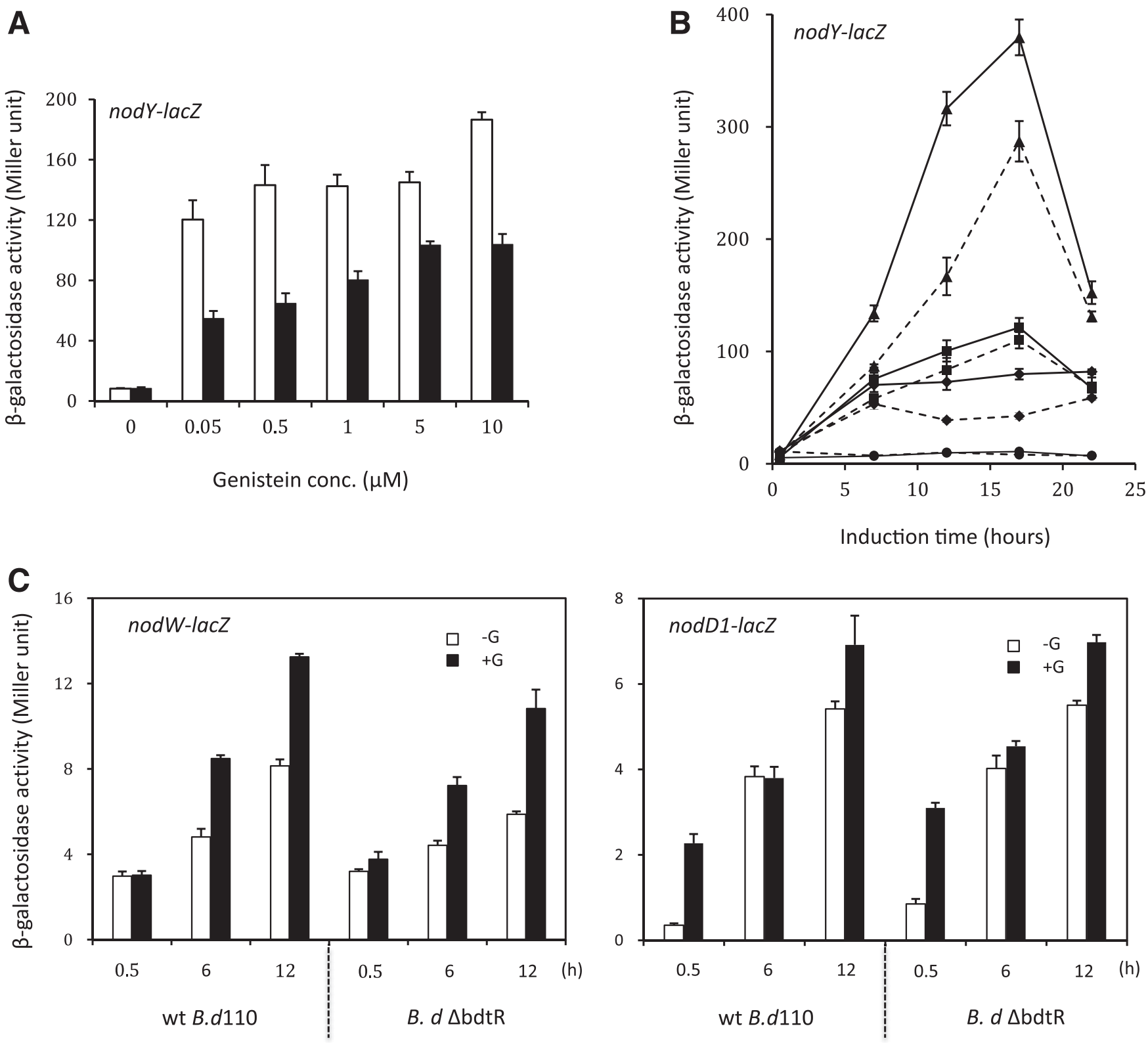

Fig. 7. A, nodY-lacZ activities induced by varying concentrations of genistein in the background of wild-type (WT) Bradyrhizobium diazoefficiens 110 (white column) and $B$. diazoefficiens $\Delta$ bdtR (black column) for $12 \mathrm{~h}$ postinduction. B, Time course of nodY-lac $Z$ activity in response to $5.0 \mu \mathrm{M}$ genistein (triangles), $5.0 \mu \mathrm{M}$ daidzein (squares), and $20 \mu \mathrm{l}$ of culture soybean seed extract per milliliter (diamonds) in the background of strains WT B. diazoefficiens 110 (solid line) and $B$. diazoefficiens $\Delta \mathrm{bdtR}$ (dotted line). Dots indicate the blank control, only pure EtOH. nodY-lacZ translational fusion was carried out in the pZB32 plasmid. C, nodW-and nodD1-lacZ activities in WT B. diazoefficiens 110 (wt B.d110) and B. diazoefficiens $\Delta$ bdtR (B. $d \Delta$ bdtR) in response to 5.0 $\mu \mathrm{M}$ genistein. Chromosomally integrated fusions of transcriptional nodW-and nodD1-lac $Z$ were generated in this study. $-\mathrm{G}$ and $+\mathrm{G}$ indicate the absence and presence of genistein, respectively. Error bars indicate the standard error of the mean of three independent experiments. 
plant-released (iso)flavonoids that are encountered in the early stage of soybean-Bradyrhizobium diazoefficiens symbiotic development.

\section{MATERIALS AND METHODS}

\section{Bacterial strains and culture conditions.}

The bacterial strains and plasmids used in this study are shown in Table 1. Bradyrhizobium diazoefficiens was grown in yeast extract-mannitol (YEM) medium at $30^{\circ} \mathrm{C}$, and the Escherichia coli strain was maintained in Luria-Bertani medium at $37^{\circ} \mathrm{C}$. Arabinose-gluconate medium and HM salt medium supplemented with $0.1 \%$ (wt/vol) arabinose (Cole and Elkan 1973; Sadowsky et al. 1987) were used for bi- or triparental mating. The media were supplemented with appropriate antibiotics at the following concentrations (per milliliter): for $B$. diazoefficiens: chloramphenicol $(30 \mu \mathrm{g})$, spectinomycin $(200 \mu \mathrm{g})$, streptomycin $(200 \mu \mathrm{g})$, tetracycline $(100 \mu \mathrm{g})$, and kanamycin $(100 \mu \mathrm{g})$; for E. coli: kanamycin $(50 \mu \mathrm{g})$, streptomycin $(50 \mu \mathrm{g})$, spectinomycin $(50 \mu \mathrm{g})$, and tetracycline $(10 \mu \mathrm{g})$. Genistein and daidzein were purchased from Solarbio Life Sciences Co., Ltd., Beijing), and SSE was produced as previously described (Wei et al. 2008).

\section{Construction of the $b d t R$ mutant, transcriptional lacZ fusions, and GusA-marked strains.}

The insertional and in-frame deletion mutant strains $B$. diazoefficiens bdtR and $B$. diazoefficiens $\Delta$ bdtR were generated according to the detailed methods described in Supplementary Text $\mathrm{S} 1$. In brief, the $b d t R$ gene of the former strain was mutated via the replacement of an antibiotic $S \mathrm{~m} / \mathrm{Spc} \Omega$ cassette, and a 390-nt-long region was deleted from that of the latter strain. For the transcriptional lacZ fusions, bll7022- and $b d t R-l a c Z$ were generated in plasmid pKS801, which was a

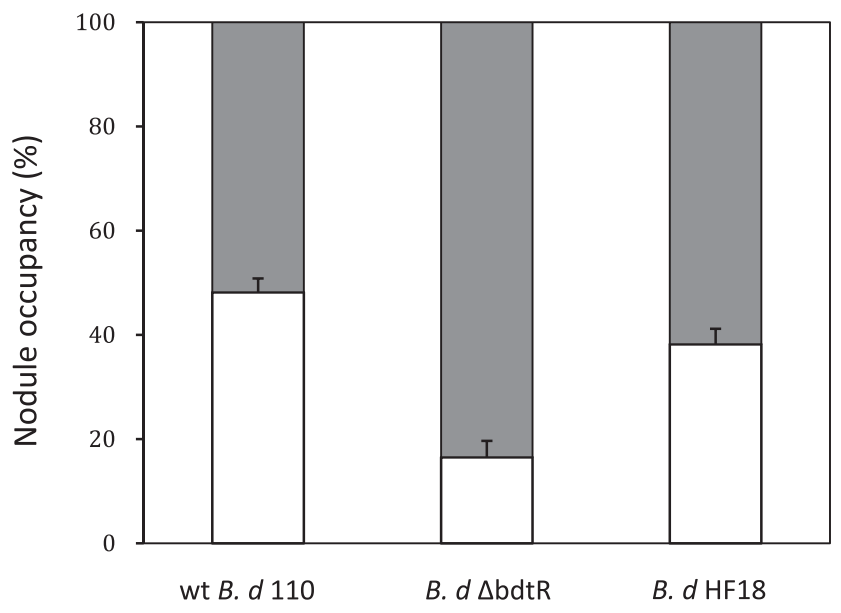

Test strains

$$
\text { B. } d \mathrm{~A} 20
$$

test strains

Fig. 8. Competitive nodule occupancies of test strains (wild type Bradyrhizobium diazoefficiens $110(B . d 110), B$. diazoefficiens $\Delta \mathrm{bdtR}(B$. $d \Delta \mathrm{bdtR}$ ), and the complemented strain B. diazoefficiens HF18 (B. $d \mathrm{HF} 18)$ upon co-inoculation with the GusA-marked wild-type strain (B. diazoefficiens A20). Each test strain was mixed with the B. diazoefficiens A20 strain $(B . d \mathrm{~A} 20)$ at a ratio of 1:1 and was inoculated onto soybean seeds. Five weeks after inoculation, the nodules of 25 plants in each treatment were stained with $\beta$-glucuronidase and numbered. The experiment was performed three times, and the data are presented as the means \pm standard deviations. In each independent experiment, the $\Delta \mathrm{bdtR}$ mutant was less competitive than the A20 strain $(P<0.01)$ product of the broad-host-range plasmid pKS800, while the nodD1 - and nodW-lacZ fusions were generated through chromosomal integration, using the suicide plasmid pVIK112 containing a promoterless $l a c Z$ gene (Supplementary Text S1). GusA-marked WT B. diazoefficiens 110 (B. diazoefficiens A20) was constructed by biparental mating with E. coli S17-1 $\lambda$ pir carrying the pCAM120 plasmid as a donor strain. The conjugant was tested for its $\beta$-glucuronidase (GUS) activity with 5bromo-4-chloro-3-indolyl- $\beta$-D-glucuronide (X-Gluc) $(100 \mu \mathrm{g} / \mathrm{ml})$ as the substrate and was subsequently screened based on showing nodulation and nitrogen-fixing abilities similar to those of WT B. diazoefficiens 110.

\section{Determination of $\boldsymbol{\beta}$-galactosidase activity.}

Induction was carried out in $3 \mathrm{ml}$ of YEM culture diluted to $\mathrm{OD}_{600}=0.1$. A total of $250 \mu \mathrm{l}$ of the cell suspension was mixed with $200 \mu \mathrm{l}$ of Z-buffer and was lysed with $40 \mu \mathrm{l}$ of chloroform and $40 \mu \mathrm{l}$ of $0.1 \%$ sodium dodecyl sulfate (SDS) at $30^{\circ} \mathrm{C}$ for $10 \mathrm{~min}$. $\beta$-Galactosidase activity was measured by taking a $150-\mu l$ sample of the permeabilized cell suspension and mixing it with $600 \mu \mathrm{l}$ of a solution containing $4 \mathrm{mg} \mathrm{ml}^{-1} O$ nitrophenyl- $\beta$-D-galactoside (Solarbio Life Sciences Co., Ltd.). After incubation for $2 \mathrm{~h}$ at room temperature, the reaction was interrupted by the addition of $750 \mu \mathrm{l}$ of $0.5 \mathrm{M} \mathrm{Na}_{2} \mathrm{CO}_{3}$. One unit of $\beta$-galactosidase activity was expressed as the millimoles of $o$-nitrophenol liberated per minute according to the absorption changes at $\mathrm{OD}_{420}$ on the basis of the cell population density $\left(\mathrm{OD}_{600}\right)$.

\section{Heterologous expression of BdtR as}

a glutathione- $S$-transferase (GST) fusion protein.

$b d t R$ was amplified by PCR, using the primers blr7023for_ EcoR I and blr7023rev_SalI, and was cloned into the vector pGEX4-T3, resulting in the pbdtR plasmid, which was transformed into BL21(DE3). The pbdtR insert was verified by sequencing. For the purification of the BdtR protein, expression was induced for $3 \mathrm{~h}$ by isopropyl- $\beta$-D-thiogalactoside (300 $\mu \mathrm{M})$. Cells from a $100-\mathrm{ml}$ culture were harvested and lysed at a pressure of $27 \mathrm{kpsi}$ (Constant Systems Limited, Low March, Northampton, U.K.). The BdtR protein was purified using protein G(A) agarose 4 FF sepharose (AOGMA, Xuhui District, Shanghai, China). GST was removed by on-column cleavage with thrombin according to supplier protocol (Roche, Pudong District, Shanghai, China).

\section{EMSA.}

The 213-bp-long PCR product encompassing the intervening region of bll7022-bdtR was generated and fluorescently labeled with Cy5 dye, using primers AirF and AirR (Table 1) (Sangong Corp., Shanghai, China). BdtR was incubated with the Cy5labeled PCR product in binding buffer $(100 \mathrm{mM}$ Tris, $25 \%$ glycerol, $0.2 \mu \mathrm{g}$ of bovine serum albumin per microliter, $50 \mu \mathrm{M}$ $\mathrm{MgCl}_{2}, 5 \mu \mathrm{M}$ dithiothreitol, $\mathrm{pH} 7.9$ ) at $30^{\circ} \mathrm{C}$ for $20 \mathrm{~min}$. Then, the reaction mixture was subjected to electrophoresis at $4^{\circ} \mathrm{C}$ in a $6 \%$ native polyacrylamide gel in $0.5 \times$ Tris-borate-EDTA buffer (44.5 mM Tris, $44.5 \mathrm{mM}$ boric acid, $2 \mathrm{mM}$ EDTA, $\mathrm{pH}$ 8.3 ) for 1.5 to $2 \mathrm{~h}$ at $100 \mathrm{~V}$. Fluorescently labeled DNA in the gel was detected with a Starion FLA-9000 system (FujiFilm, Tokyo).

\section{Determination of transcriptional start sites by $5^{\prime}$-RACE.}

WT B. diazoefficiens 110 cells were harvested $0.5 \mathrm{~h}$ after induction with $5 \mu \mathrm{M}$ genistein. Total RNA was isolated as described previously (Wei et al. 2008). RT was carried out with Superscript II RT (Invitrogen, Carlsbad, CA, U.S.A.) at $42^{\circ} \mathrm{C}$ for $50 \mathrm{~min}$, using the gene-specific primer GSP1 (Table 1). The resulting cDNA was treated with RNase $\mathrm{H}$ at $37^{\circ} \mathrm{C}$ for $30 \mathrm{~min}$ 
and was purified with GlassMAX DNA isolation spin cartridges (Life Technologies, Waltham, MA, U.S.A.). dC tails were added to the $3^{\prime}$ end of the cDNA using terminal deoxynucleotidyl transferase. The double-stranded DNA was amplified with the abridged anchor primer and a second genespecific primer, GSP2 (Table 1). Then, an aliquot of the obtained PCR product served as the template for semi-nested PCR using an abridged universal amplification primer and a

Table 1. Bacterial strains and plasmids used in the study

\begin{tabular}{|c|c|c|}
\hline Strains or plasmids & Characteristics or sequence $^{a}$ & Reference or source \\
\hline \multicolumn{3}{|l|}{ Bradyrhizobium diazoefficiens } \\
\hline USDA 110 & Wild type (WT), $\mathrm{Cm}^{\mathrm{r}}$ & $\begin{array}{l}\text { United States Department of } \\
\text { Agriculture, Beltsville, MD, U.S.A. }\end{array}$ \\
\hline$\triangle \mathrm{RND}$ & Deletion of bll7019-bll7021 of USDA110/insertion of $\Omega, \mathrm{Sm}^{\mathrm{r}} ; \mathrm{Sp}^{\mathrm{r}}$ & Takeshima et al. 2013 \\
\hline bdtR & Insertion of blr7023 of USDA110/insertion of $\Omega, \mathrm{Sm}^{\mathrm{r}} ; \mathrm{Sp}^{\mathrm{r}}$ & This study \\
\hline$\Delta \mathrm{bdtR}$ & In-frame deletion of blr7023 with $390 \mathrm{bp}, \mathrm{Cm}^{\mathrm{r}}$ & This study \\
\hline $\mathrm{A} 20$ & WT B. diazoefficiens USDA 110 , inserted with Tn5::gusA, $\mathrm{Cm}^{\mathrm{r}} ; \mathrm{Sp}^{\mathrm{r}}$ & This study \\
\hline HF18 & B. diazoefficiens bdt $\mathrm{R}$ mutant strain carrying $\mathrm{pKS} 811, \mathrm{Tc}^{\mathrm{r}}$ & This study \\
\hline HQ30 & $\begin{array}{l}\text { Carrying plasmid pKS818 containing a bdtR-lac Z transcriptional fusion, } \\
\mathrm{Tc}^{\mathrm{r}}\end{array}$ & This study \\
\hline HQ31 & $\begin{array}{l}\text { Carrying plasmid pKS819 containing a bll7022-lacZ transcriptional fusion, } \\
\mathrm{Tc}^{\mathrm{r}}\end{array}$ & This study \\
\hline $\mathrm{BX} 2$ & WT B. diazoefficiens 110 carrying plasmid pZB32, $\mathrm{Tc}^{\mathrm{r}}$ & This study \\
\hline BX3 & Mutant strain B. diazoefficiens $\Delta$ bdtR carrying plasmid pZB32, $\mathrm{Tc}^{\mathrm{r}}$ & This study \\
\hline \multicolumn{3}{|l|}{ Escherichia coli } \\
\hline S17-1 $\lambda$-pir & TpRSmRrecA, thi, pro, hsdR-M+RP4: 2-Tc:Mu: Km Tn7 $\lambda$-pir & Metcalf et al. 1994 \\
\hline DH5 $\alpha$ & 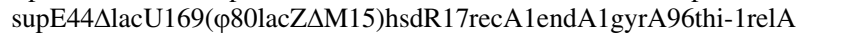 & Thermo Fisher Scientific Inc. \\
\hline BL21(DE3) & $\mathrm{F}^{-}$ompT hsd $S_{\mathrm{R}}\left(\mathrm{r}_{\mathrm{R}}^{-} \mathrm{m}_{\mathrm{R}}^{-}\right)$gal dcm $\lambda(\mathrm{DE} 3)$ & Invitrogen \\
\hline \multicolumn{3}{|l|}{ Plasmid } \\
\hline pKS800 & Derivative of the broad-host-range cosmid pLAFR1, IncP Tc ${ }^{r}$ & Hattori et al. 2002 \\
\hline pVIK112 & Plasmid carrying promoterless lacZYA genes, $\mathrm{Km}^{\mathrm{r}}$ & Kalogeraki and Winans 1997 \\
\hline pK18mobSacB & Mobilizable suicide vector, $\mathrm{Km}^{\mathrm{r}}$ & Schäfer et al. 1994 \\
\hline pHP45 2 & Plasmid carrying an $\Omega$ cassette, $\mathrm{Sm}^{\mathrm{r}} ; \mathrm{Sp}^{\mathrm{r}}$ & Prentki and Krisch 1984 \\
\hline pRK2013 & ColE1 replicon carrying $\mathrm{RK} 2$ transfer genes, $\mathrm{Km}^{\mathrm{r}}$ & Figurski and Helinski 1979 \\
\hline pCAM 120 & pmTn5SSgusA20, mini-Tn 5 transposon containing an $\Omega$ cassette, $\mathrm{Sp}^{\mathrm{r}}$; gusA & Wilson et al. 1995 \\
\hline pGEX-4T-3 & Vector for generation of $g s t$ fusions, $\mathrm{Ap}^{\mathrm{r}}$ & GE Healthcare, Chalfont St.Giles, U.K. \\
\hline pMD18T & $\begin{array}{l}\text { Vector for cloning and sequencing of the final amplification product of } 5^{\prime} \\
\text { rapid amplification of cDNA ends }\end{array}$ & Takara Co. \\
\hline pZB32 & nodY-lacZ translational fusion on a broad-host-range plasmid pPP375, $\mathrm{Tc}^{\mathrm{r}}$ & Banfalvi et al. 1988 \\
\hline pbdtR & pGEX-4T-3 containing a 663-bp fragment of blr7023, Ap ${ }^{\mathrm{r}}$ & This study \\
\hline pKS811 & $\begin{array}{l}\text { pKS800 carrying a 3.0-kb fragment around wild allelic gene } b d t R \text { in the } \\
\text { PmeI site, } \mathrm{Tc}^{\mathrm{r}}\end{array}$ & This study \\
\hline pKS801 & $\begin{array}{l}\text { pKS800 with an insertion of a promoterless lacZYA fragment from } \\
\text { pVIK112 in the XhoI site, } \mathrm{Tc}^{\mathrm{r}}\end{array}$ & This study \\
\hline pKS818 & $\begin{array}{l}\text { A DNA fragment of intergenic region between bllr7022 and } b d t R \text { and } \\
\text { partial } 5^{\prime} \text { end of bll7022 was inserted in the upstream of lacZYA of } \\
\text { pKS } 801, \mathrm{Tc}^{\mathrm{r}}\end{array}$ & This study \\
\hline pKS819 & $\begin{array}{l}\text { A DNA fragment of intergenic region between bllr7022 and blr7023 and } \\
\text { partial 5' end of blr7023 was inserted in the upstream of lacZYA of } \\
\text { pKS801, } \mathrm{Tc}^{\mathrm{r}}\end{array}$ & This study \\
\hline \multicolumn{3}{|c|}{ 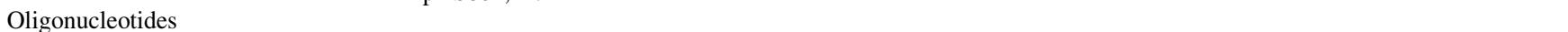 } \\
\hline bll7022_GSP1 & GACCCTGTGATGTAAC & Transcript mapping \\
\hline bll7022_GSP2 & AGCAGGATGCCGTGTTGT & Transcript mapping \\
\hline bll7022_GSP3 & CGGTCCGGGTCGGGGCA & Transcript mapping \\
\hline$b d t R \_\mathrm{GSP} 1$ & CGAGAAAAAGCTGCTG & Transcript mapping \\
\hline$b d t R \_G S P 2$ & GGCTGCGTCAAGGATC & Transcript mapping \\
\hline$b d t R \_$GSP3 & GCAAGGTCTTTGGTCGGT & Transcript mapping \\
\hline abridged anchor primer & GGCCACGCGTCGACTAGTACGGGGGGGGGG & Transcript mapping \\
\hline $\begin{array}{l}\text { abridged universal amplification } \\
\text { primer }\end{array}$ & GGCCACGCGTCGACTAGTAC & Transcript mapping \\
\hline pVIK112_laZYA_F & *TCCGTTTAAACTCGAAGAGCTCGGTACCTCTAGAT & Construction of pKS801 \\
\hline pVIK112_lacZYA_R & *CGCTGAATTACTCGAAACCGCCCAGTCTAGCTATCG & Construction of pKS801 \\
\hline blr7023for_EcoRI & GGAATTCCATGACCAAGAAGAGCACCCCG & Generation of gst-bdtR fusion \\
\hline blr7023rev_Sal I & A $\overline{C G C G T C G A C T C A C G G C G T C C A G T C T G C C T C ~}$ & Generation of gst-bdtR fusion \\
\hline AirF & **cy5-AGCCAGTGGCGATAAGTATTCGCTCTCTCCTGAGGTCG & $\begin{array}{l}\text { Amplification of intergenic region } \\
\text { between } b l l 7022 \text { and } \text { blr } 7023\end{array}$ \\
\hline AirR & **cy5-AGCCAGTGGCGATAAGTCCGTCGATCCTCCAGTCAC & $\begin{array}{l}\text { Amplification of intergenic region } \\
\text { between } b l l 7022 \text { and } b l r 7023\end{array}$ \\
\hline CbdtR_F & *GAATTACTCGAGTTTCGCTCAGTTCGACCGATTTT & Generation of pKS 811 \\
\hline CbdtR_R & *ATTAAGGATCCGTTTCAGGCAAAGAAAGTCGGACC & Generation of pKS811 \\
\hline
\end{tabular}

${ }^{a} \mathrm{Ap}^{\mathrm{r}}, \mathrm{Cm}^{\mathrm{r}}, \mathrm{Km}^{\mathrm{r}}, \mathrm{Sm}^{\mathrm{r}}, \mathrm{Sp}^{\mathrm{r}}$, and $\mathrm{Tc}^{\mathrm{r}}$ indicate ampicillin, chloramphenicol, kanamycin, streptomycin, spectinomycin, and tetracycline resistance, respectively. One asterisk (*) indicates the underlined oligonucleotides comprise the homology sequence flanking the XhoI and PmeI of pKS800, respectively. Two asterisks $(* *)$ indicate the underlined oligonucleotides comprise the cy5-labeled universal primer. Double-underlined oligonucleotides indicate the sequence of corresponding restriction enzymes for cloning. Bold formatting indicates a putative sequence of the AT-rich Shine Dagarno-like sequence of pVIK112. 
third gene-specific primer, GSP3 (Table 1). The resulting PCR products were cloned into pMD18T and were sequenced. The transcriptional site was considered validated if the longest sequence was obtained with at least five independent clones.

\section{Macroarray analysis and quantitative real-time RT-PCR.}

The macroarray platform was constructed and expression was analyzed as described previously. Approximately $80 \mathrm{ng}$ of total RNA was used as template, and real-time RT-PCR was performed in an ABI 7500 fast system (Thermo Fisher Scientific Inc., Waltham, MA, U.S.A.) with the One Step TB Green PrimeScript RT-PCR kit (Takara Co., Ltd., Beijing). Quantification was performed using QuantStudio design and analysis software (Thermo Fisher Scientific Inc.) according to the application guide. The $B$. diazoefficiens housekeeping gene sigA, which showed no significant change in expression levels under the growth conditions applied in this experiment, was used as an internal reference.

\section{Nodulation competitiveness test.}

The strains were cultured in YEM containing the corresponding antibiotics at $30^{\circ} \mathrm{C}$ for 6 days. Cells were washed twice with sterile saline and were suspended in sterile water; the cell density of the suspension was then measured, and the suspension was diluted to $10^{7}$ cells per milliliter. The suspended cells of WT B. diazoefficiens $110, B$. diazoefficiens $\Delta \mathrm{bdtR}$, and $B$. diazoefficiens HF18 were individually mixed with the GusAmarked wild-type strain (B. diazoefficiens A20) at a ratio of 1:1. One milliliter of the suspension was inoculated onto the seeds of Glycine max cv. Suinong4, which were surface-sterilized according to the commonly described process. Twenty-five plants were used for each treatment and were grown in sterilized vermiculite, which was watered with $20 \mathrm{ml}$ of nitrogenfree plant nutrient solution (Norris and Date 1976) under a 14-h day and 10-h night light regime. After inoculation for 35 days, the nodules were harvested for the GUS assay as follows. Roots attached to the nodules were immersed in GUS reaction solution $(20 \mathrm{mg}$ of X-Gluc per liter, $0.04 \%$ SDS, $20 \%$ methanol, $20 \mathrm{mM}$ sodium phosphate buffer, $\mathrm{pH}$ 7.0), were exposed to vacuum for $15 \mathrm{~min}$, and were incubated at $30^{\circ} \mathrm{C}$ overnight. Then, the stained nodules were counted.

\section{Analysis of extracellular genistein concentration.}

The genistein concentration in the supernatant was measured as follows. The cells of three strains grown to $\mathrm{OD}_{600}=$ 0.4 to 0.6 were individually diluted to $\mathrm{OD}_{600}=0.1$ with fresh YEM in a total volume of $100 \mathrm{ml}$, and genistein was added to a final concentration of $10.0 \mu \mathrm{M}$. After different incubation periods, the genistein content in the supernatant was measured by high-performance liquid chromatography (HPLC). HPLC was performed with a Dionex UltiMate 3000 system under the following conditions: column, Waters Symmetry C18 $5.0 \mu \mathrm{m}(4.6 \times 250 \mathrm{~mm})$; mobile phase, methanol/water (70:30); flow rate, $0.6 \mathrm{ml} / \mathrm{min}$; temperature, $25^{\circ} \mathrm{C}$; detection, $262 \mathrm{~nm}$. The total cellular protein content was determined with a Bradford kit (Solarbio Life Sciences Co., Ltd.) for normalization.

\section{ACKNOWLEDGMENTS}

We greatly thank M. Göttfert for helping the bioinformatic analysis of the palindromic sequence.

\section{LITERATURE CITED}

Aendekerk, S., Diggle, S. P., Song, Z., Høiby, N., Cornelis, P., Williams, P., and Cámara, M. 2005. The MexGHI-OpmD multidrug efflux pump controls growth, antibiotic susceptibility and virulence in Pseudomonas aeruginosa via 4-quinolone-dependent cell-to-cell communication. Microbiol. Read. 151:1113-1125.

Banfalvi, Z., Nieuwkoop, A., Schell, M., Besl, L., and Stacey, G. 1988. Regulation of nod gene expression in Bradyrhizobium japonicum. Mol. Gen. Genet. 214:420-424.

Cole, M. A., and Elkan, G. H. 1973. Transmissible resistance to penicillin G, neomycin, and chloramphenicol in Rhizobium japonicum. Antimicrob. Agents Chemother. 4:248-253.

Dobrindt, U., Hochhut, B., Hentschel, U., and Hacker, J. 2004. Genomic islands in pathogenic and environmental microorganisms. Nat. Rev. Microbiol. 2:414-424.

Eda, S., Mitsui, H., and Minamisawa, K. 2011. Involvement of the $s m e A B$ multidrug efflux pump in resistance to plant antimicrobials and contribution to nodulation competitiveness in Sinorhizobium meliloti Appl. Environ. Microbiol. 77:2855-2862.

Figurski, D. H., and Helinski, D. R. 1979. Replication of an origincontaining derivative of plasmid RK2 dependent on a plasmid function provided in trans. Proc. Natl. Acad. Sci. U.S.A. 76:1648-1652.

Gao, M., Teplitski, M., Robinson, J. B., and Bauer, W. D. 2003. Production of substances by Medicago truncatula that affect bacterial quorum sensing. Mol. Plant-Microbe Interact. 16:827-834.

González, J. E., and Keshavan, N. D. 2006. Messing with bacterial quorum sensing. Microbiol. Mol. Biol. Rev. 70:859-875.

González-Pasayo, R., and Martínez-Romero, E. 2000. Multiresistance genes of Rhizobium etli CFN42. Mol. Plant-Microbe Interact. 13: 572-577.

Göttfert, M., Grob, P., and Hennecke, H. 1990. Proposed regulatory pathway encoded by the nodV and nodW genes, determinants of hos specificity in Bradyrhizobium japonicum. Proc. Natl. Acad. Sci. U.S.A. $87: 2680-2684$.

Göttfert, M., Lamb, J. W., Gasser, R., Semenza, J., and Hennecke, H. 1989. Mutational analysis of the Bradyrhizobium japonicum common nod genes and further nod box-linked genomic DNA regions. Mol. Gen. Genet. 215:407-415.

Graham, T. L. 1991. Flavonoid and isoflavonoid distribution in developing soybean seedling tissues and in seed and root exudates. Plant Physiol. 95: 594-603.

Hattori, Y., Omori, H., Hanyu, M., Kaseda, N., Mishima, E., Kaneko, T., Tabata, S., and Saeki, K. 2002. Ordered cosmid library of the Mesorhizobium loti MAFF303099 genome for systematic gene disruption and complementation analysis. Plant Cell Physiol. 43:1542-1557.

Kalogeraki, V. S., and Winans, S. C. 1997. Suicide plasmids containing promoterless reporter genes can simultaneously disrupt and create fusions to target genes of diverse bacteria. Gene 188:69-75

Kaneko, T., Nakamura, Y., Sato, S., Minamisawa, K., Uchiumi, T. Sasamoto, S., Watanabe, A., Idesawa, K., Iriguchi, M., Kawashima, K., Kohara, M., Matsumoto, M., Shimpo, S., Tsuruoka, H., Wada, T., Yamada, M., and Tabata, S. 2002. Complete genomic sequence of nitrogen-fixing symbiotic bacterium Bradyrhizobium japonicum USDA110. DNA Res. 9:189-197.

Kosslak, R. M., Bookland, R., Barkei, J., Paaren, H. E., and Appelbaum, E. R. 1987. Induction of Bradyrhizobium japonicum common nod genes by isoflavones isolated from Glycine max. Proc. Natl. Acad. Sci. U.S.A. 84:7428-7432.

Krummenacher, P., and Narberhaus, F. 2000. Two genes encoding a putative multidrug efflux pump of the RND/MFP family are cotranscribed with an rpoH gene in Bradyrhizobium japonicum. Gene 241:247-254.

Lindemann, A., Koch, M., Pessi, G., Müller, A. J., Balsiger, S., Hennecke, H., and Fischer, H. M. 2010. Host-specific symbiotic requirement of BdeAB, a RegR-controlled RND-type efflux system in Bradyrhizobium japonicum. FEMS Microbiol. Lett. 312:184-191.

Loh, J., Garcia, M., and Stacey, G. 1997. NodV and NodW, a second flavonoid recognition system regulating nod gene expression in Bradyrhizobium japonicum. J. Bacteriol. 179:3013-3020.

Loh, J., Lohar, D. P., Andersen, B., and Stacey, G. 2002. A two-component regulator mediates population-density-dependent expression of the Bradyrhizobium japonicum nodulation genes. J. Bacteriol. 184: 1759-1766.

Martinez, J. L., Sánchez, M. B., Martínez-Solano, L., Hernandez, A., Garmendia, L., Fajardo, A., and Alvarez-Ortega, C. 2009. Functional role of bacterial multidrug efflux pumps in microbial natural ecosystems. FEMS Microbiol. Rev. 33:430-449.

Metcalf, W. W., Jiang, W., and Wanner, B. L. 1994. Use of the rep technique for allele replacement to construct new Escherichia coli hosts for maintenance of R6K $\gamma$ origin plasmids at different copy numbers. Gene 138:1-7.

Neyfakh, A. A. 1997. Natural functions of bacterial multidrug transporters. Trends Microbiol. 5:309-313. 
Nikaido, H. 1996. Multidrug efflux pumps of gram-negative bacteria. J. Bacteriol. 178:5853-5859.

Norris, D. O., and Date, R. A. 1976. Legume bacteriology, tropical pasture principles and methods. CAB Bulletin 51:134-147.

Palumbo, J. D., Kado, C. I., and Phillips, D. A. 1998. An isoflavonoidinducible efflux pump in Agrobacterium tumefaciens is involved in competitive colonization of roots. J. Bacteriol. 180:3107-3113.

Parniske, M., Ahlborn, B., and Werner, D. 1991. Isoflavonoid-inducible resistance to the phytoalexin glyceollin in soybean rhizobia. J. Bacteriol. 173:3432-3439.

Piddock, L. J. 2006. Multidrug-resistance efflux pumps-Not just for resistance. Nat. Rev. Microbiol. 4:629-636.

Prentki, P., and Krisch, H. M. 1984. In vitro insertional mutagenesis with a selectable DNA fragment. Gene 29:303-313.

Rossbach, S., Kunze, K., Albert, S., Zehner, S., and Göttfert, M. 2014. The Sinorhizobium meliloti EmrAB efflux system is regulated by flavonoids through a TetR-like regulator (EmrR). Mol. Plant-Microbe Interact. 27: 379-387.

Sadowsky, M. J., Tully, R. E., Cregan, P. B., and Keyser, H. H. 1987. Genetic diversity in Bradyrhizobium japonicum serogroup 123 and its relation to genotype-specific nodulation of soybean. Appl. Environ. Microbiol. 53:2624-2630.

Saier, M. H. J., Jr., and Paulsen, I. T. 2001. Phylogeny of multidrug transporters. Semin. Cell Dev. Biol. 12:205-213.

Schäfer, A., Tauch, A., Jäger, W., Kalinowski, J., Thierbach, G., and Pühler, A. 1994. Small mobilizable multi-purpose cloning vectors derived from the Escherichia coli plasmids pK18 and pK19: Selection of defined deletions in the chromosome of Corynebacterium glutamicum. Gene 145:69-73.

Schlaman, H. R. M., Phillips, D. A., and Kondorosi, E. 1998. Genetic organization and transcriptional regulation of rhizobial nodulation genes.
Pages 361-386 in: The Rhizobiaceae. H. Spaink, A. Kondorosi, and P. J. J. Hooykaas, eds. Kluwer Academic Publisher, Dordrecht, Netherlands. https://doi.org/10.1007/978-94-011-5060-6_19

Sekiya, H., Mima, T., Morita, Y., Kuroda, T., Mizushima, T., and Tsuchiya, T. 2003. Functional cloning and characterization of a multidrug efflux pump, mexHI-opmD, from a Pseudomonas aeruginosa mutant. Antimicrob. Agents Chemother. 47:2990-2992.

Solovyev, V., and Salamov, A. 2011. Automatic annotation of microbial genomes and metagenomic sequences. Pages 61-78 in: Metagenomics and Its Applications in Agriculture, Biomedicine and Environmental Studies. R. W. Li, ed. Nova Science Publishers, Hauppauge, NY, U.S.A

Takeshima, K., Hidaka, T., Wei, M., Yokoyama, T., Minamisawa, K., Mitsui, H., Itakura, M., Kaneko, T., Tabata, S., Saeki, K., Oomori, H., Tajima, S., Uchiumi, T., Abe, M., Tokuji, Y., and Ohwada, T. 2013. Involvement of a novel genistein-inducible multidrug efflux pump of Bradyrhizobium japonicum early in the interaction with Glycine max (L.) Merr. Microbes Environ. 28:414-421.

Wei, M., Yokoyama, T., Minamisawa, K., Mitsui, H., Itakura, M., Kaneko, T., Tabata, S., Saeki, K., Omori, H., Tajima, S., Uchiumi, T., Abe, M., and Ohwada, T. 2008. Soybean seed extracts preferentially express genomic loci of Bradyrhizobium japonicum in the initial interaction with soybean, Glycine $\max (\mathrm{L}$.$) Merr. DNA Res. 15:201-214.$

Wenzel, M., Lang, K., Günther, T., Bhandari, A., Weiss, A., Lulchev, P., Szentgyörgyi, E., Kranzusch, B., and Göttfert, M. 2012. Characterization of the flavonoid-responsive regulator FrrA and its binding sites. J. Bacteriol. 194:2363-2370.

Wilson, K. L., Sessitsch, A., Corbo, J. C., Giller, K. E., Akkermans, A. D., and Jefferson, R. A. 1995. $\beta$-Glucuronidase (GUS) transposons for ecological and genetic studies of rhizobia and other gram-negative bacteria. Microbiology 141:1691-1705. 